This heat-resisting stainless steel blanket fitted to the exhaust unit of the Avon engine is also provided with a gold coating to reduce heat transfer

consisting of two layers of dimpled stainless steel with a filling of silicone fibre glass-was also treated in the same way and with equal success.

This application of the low emissivity property of gold has thus been completely successful in resolving overheating problems without the need for the extensive use of insulating materials.

In addition to this application in the "Lightning" jet aircraft, these gold coatings are being employed to reduce heat transfer on dragchute containers, tail cone assemblies and missile blast shields.

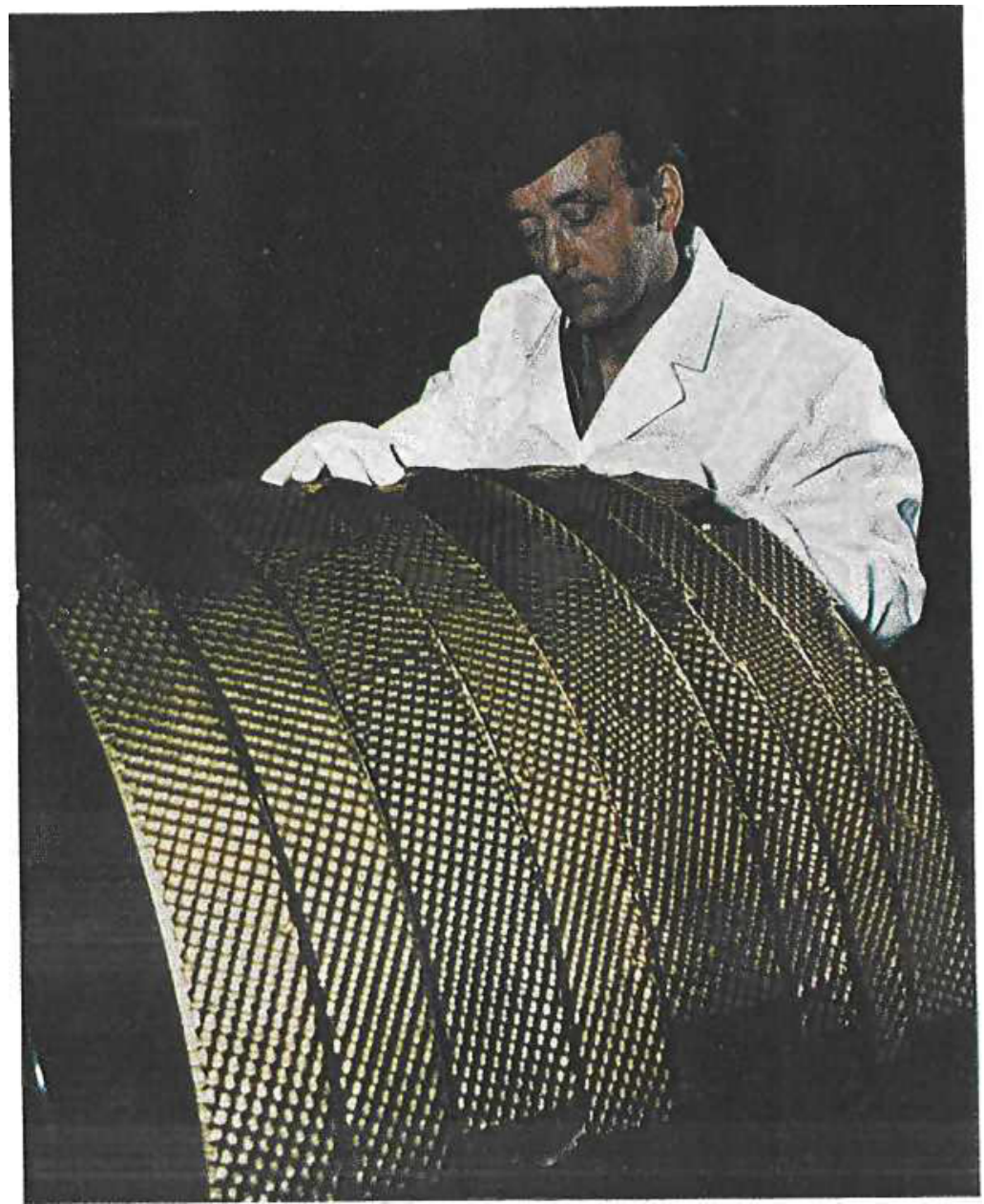

\title{
Methods of Reducing Porosity in Thin Gold Electrodeposits
}

The British Non-Ferrous Metals Research Association has recently been studying methods by which the residual porosity in thin gold electrodeposits may be reduced. Gold plating for electronic applications has to be reliably pore-free to ensure that even slight corrosion of the substrate cannot occur, particularly in the areas near contacts. A sufficient thickness of gold is therefore applied to ensure a very low density of pores.

The investigation has concentrated upon techniques which could be applied without undue difficulty to current plating procedures, and areas have been identified where an alteration in processing can result in a reduction in residual porosity. Two variables have been shown to be important in the consistent production of low porosity gold coatings. These are the original condition of the substrate to be plated and the method of agitation used during the electrodeposition process.

Surface features present on the substrate have been shown to affect significantly the porosity level of the electrodeposited gold coating. Inclusions have been identified as a source of residual porosity, but these can be removed by suitable cleaning processes. However, the surface layer of the substrate can be distorted due to working and, also, often contains depressions and craters which form a potential pore site. It has been found that the surface can be considerably improved by electropolishing prior to plating, and a procedure for producing a satisfactory substrate by electropolishing has been developed.

The effects of different methods of agitating the electrolyte during electrodeposition have also been studied. It has been found on a statistical basis that porosity can be reduced still further by utilising ultrasonic agitation during electrodeposition. A combination of the two processes, i.e. electropolishing and ultrasonic agitation, results in a consistently low residual porosity when thin $(2 \mu \mathrm{m})$ gold coatings are deposited.

It is hoped to study the causes for this slight residual porosity further and to apply these results on a larger scale in production plating shops.

I. R. A. C. 